\title{
An experimental study on the abrasive jet deburring of cross-drilled holes
}

\author{
R. Balasubramaniam ${ }^{\mathrm{a}, *}$, J. Krishnan $^{\mathrm{a}}$, N. Ramakrishnan ${ }^{\mathrm{b}}$ \\ ${ }^{a}$ Central Workshops, Bhabha Atomic Research Centre, Mumbai-85, India \\ ${ }^{\mathrm{b}}$ Department of Mechanical Engineering, Indian Institute of Technology, Mumbai-76, India
}

\begin{abstract}
Deburring of cross-drilled holes by secondary erosion of an abrasive jet was carried out and the parameters were identified. The detailed studies carried out on the parameters indicate that the parameter stand-off distance, burr root-to-stopper distance, abrasive grit size and mixing ratio significantly affect the deburring process. A burr model for $90^{\circ}$ cross-drilled holes is also presented in this paper.
\end{abstract}

Keywords: Deburring; Cross-drilled holes; Abrasive jet

\section{Introduction}

Burrs are generated in most machining processes; they cannot be completely eliminated at the machining stage, but can be minimized by controlling the process parameters. Therefore, deburring is an essential secondary operation. Deburring includes both the removal of burrs and maintainance of the proper edge condition. Deburring of external burrs is comparatively easier, owing to accessibility and the fact that the quality of the external deburring can easily be ensured by visual inspection. However, deburring of inaccessible areas poses a range of problems arising from various reasons. These include the difficulty of the deburring tool in approaching the deburring area, lack of space for positioning the deburring tool and non-visibility of the burr area. Deburring of inaccessible areas via conventional methods does not ensure burr removal and edge conditioning; deburring via non-conventional techniques provides a better solution. Electrical discharge machining (EDM), electrochemical machining (ECM) and abrasive jet machining (AJM) are some of the commonly employed non-conventional machining techniques. Of these techniques, deburring by abrasive jet $[1,2]$ is gaining increasing acceptability owing to its higher flexibility and the fact that it generates less localized force and heat at the operating area. An example of inaccessible burrs are those generated at the intersection of the cross-drilled holes. A study was carried out to establish the process parameters of the abrasive jet deburring (AJD) of cross-drilled holes, the effects of various process parameters on the deburring process and the methods necessary to maximize the material removal rate. The results of the study are presented in this paper.

In the AJM process, when accelerating abrasive particles carried by air are directed onto the target surface, the high velocity particles strike the target surface and remove the material by erosion. The mechanism of material removal is different for ductile and brittle materials. In the case of ductile materials, the erosion results from micromachining, while in the case of brittle materials, the material removal results from the intersection of lateral and radial cracks. Various investigators proposed different theories, but in general, the above-mentioned material removal mechanisms [3-7] are widely accepted.

The particles striking the target surface are reflected and some collide with the incoming particles. The remainder escape collision and travel with sufficient velocity that when another surface is struck, erosion of 
that surface results. This fact is made use of in the deburring of inaccessible areas. The burrs in inaccessible areas are difficult to remove by direct impingement of the jet, since the burrs lie over a certain area and positioning and directing the jet over this entire area is difficult. Therefore, to overcome this problem, the jet can be made to strike a primary surface on which more rebounding than erosion by the particles takes place. When these rebounding particles are directed onto the burred surface, the burrs are eroded as a result of secondary erosion.

\section{Definition of parameters}

1. Stand-off distance (SOD): this is the distance between the nozzle exit and the target surface.

2. Impingment angle(IANG): this is the angle at which the free jet strikes the target surface.

3. Burr root-to-stopper distance (BSD): this is the distance between the primary target surface and the root of the burr where the burr root thickness is maximum. Here, the primary target surface is termed as the stopper.

4. Mixing ratio (MR): this is the ratio of the weight of the abrasive to the weight of the abrasive and air per unit time.

\section{Burr model}

To study the cross-drilled hole deburring process, the generation of burr specimens was necessary. Since the quality of the deburred edges cannot be ascertained during or after deburring of the inaccessible areas, the specimens were made from split pieces which, when dismantled, exposed the cross-drilled junction. These split pieces help in studying the cross-drilled hole burrs and the deburred edges after the abrasive jet deburring process.

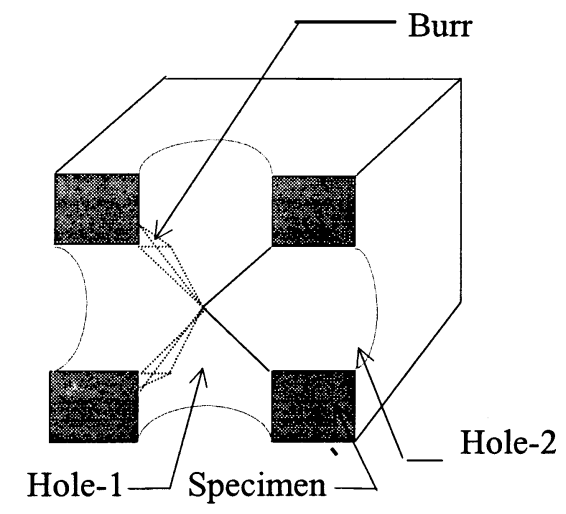

Fig. 1. Half section of burr specimen.

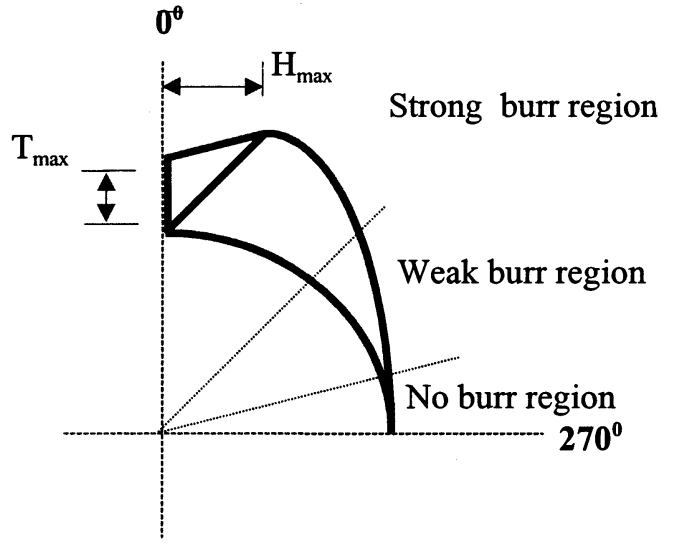

Fig. 2. Burr model.

A half-sectional view of the burr specimen used for the experiments is shown in Fig. 1. Drilling the first hole does not generate any burrs inside the specimen, but drilling the second hole generates both exit and entry burrs at the cross-hole junction. The magnitude of the exit burr at the junction is very high, while that of the entry burr is almost negligible. Therefore, for further studies only the exit burr of the second hole at the junction was considered.

Based on the measurements carried out on a number of burr specimens for burr height and burr root thickness, a burr model, as shown in Fig. 2, was constructed. The burr model is shown for the burr generated in one quadrant of the hole only. In this model, the inner surface of the burr lies on the surface of the second drilled hole. The burr height varies from $\mathrm{H}_{\max }$ at $0^{\circ}$ to $0 \mathrm{~mm}$ at $90^{\circ}$, from $0 \mathrm{~mm}$ at $90^{\circ}$ to $\mathrm{H}_{\max }$ at $180^{\circ}$, from $\mathrm{H}_{\max }$ at $180^{\circ}$ to $0 \mathrm{~mm}$ at $270^{\circ}$ and from $0 \mathrm{~mm}$ at $270^{\circ}$ to $\mathrm{H}_{\max }$ at $360^{\circ}$. The burr root thickness is maximum at $\mathrm{H}_{\max }$ and it gradually reduces to zero at the $0-\mathrm{mm}$ burr height. Some properties of the generated burr are shown in Table 1.

The burr model shown in Fig. 2 consists of three distinct regions of burr, namely the strong burr region, the weak burr region and the no-burr region.

The strong burr region is the region in which a portion of the burr is strongly attached to the specimen and where both the burr height and root thickness are maximum. In addition, a major portion of the total volume of the burr lies within this region.

Table 1

Some properties of the generated burr

\begin{tabular}{ll}
\hline Type & Exit burr \\
\hline Location & Inaccessible area \\
Maximum height & $\mathrm{H}_{\max }$ \\
Minimum height & $0 \mathrm{~mm}$ \\
Maximum root thickness & $\mathrm{T}_{\max }$ \\
Minimum root thickness & $0 \mathrm{~mm}$ \\
Toughness level & High
\end{tabular}


The weak burr region is the region in which a portion of the burr is loosely attached to the specimen and strongly attached to the burr in the strong burr region. With only a little force, the burr in this region can be detached from its root.

The no-burr region is the region in which the portion of the generated burr having minimum height and root thickness becomes detached from the specimen during the process of drilling itself.

The burrs in the no-burr region do not require a deburring operation. Those in the weak burr region require less energy to detach them from the specimen, but since they are also attached to the burrs in the strong burr region, it is essential to remove these strongly attached burrs in order to remove the weakly attached burrs. Therefore, in order to effectively implement the deburring of cross-drilled holes, burrs in the strong burr region must be completely removed.

\section{Selection of process parameters}

The AJM parameters applicable for cutting and hole generation, namely stand-off distance, abrasive size and mixing ratio, were considered for experimental purposes. Other parameters, such as nozzle pressure and nozzle dimensions were kept constant. On preliminary experiments, an additional parameter, BSD, was found to influence the deburring process and it was included for further studies. The shape of the stopper surface was also found to influence the trajectories of the reflecting particles after primary impingement. The effects of the stopper shape were studied for effective deburring and a flat stopper surface was found to be more effective; therefore, further investigations were carried out using this type of stopper.

\section{Deburring method}

Deburring of external burrs by abrasive jet was carried out by directing the jet on the burr maintaining an impingement angle of $0^{\circ}$ and a jet height of $\mathrm{h} / 2[8,9]$. Thus, the removal of burrs was due to primary erosion. Since the external burrs were easily accessible, maintaining the above-mentioned parameters for the primary erosion of burrs was possible, whereas in the case of deburring the cross-drilled holes, primary erosion of the burrs was not feasible since the burrs at the junction of the holes were not easily accessible. Therefore, the removal of burrs by secondary erosion, i.e. eroding the burrs by reflected particles, was attempted and was found to be feasible. Fig. 3 illustrates this concept of deburring by secondary erosion. Stoppers were frequently changed to minimize the errors caused owing to the change in the shape of the stopper surface by primary erosion.

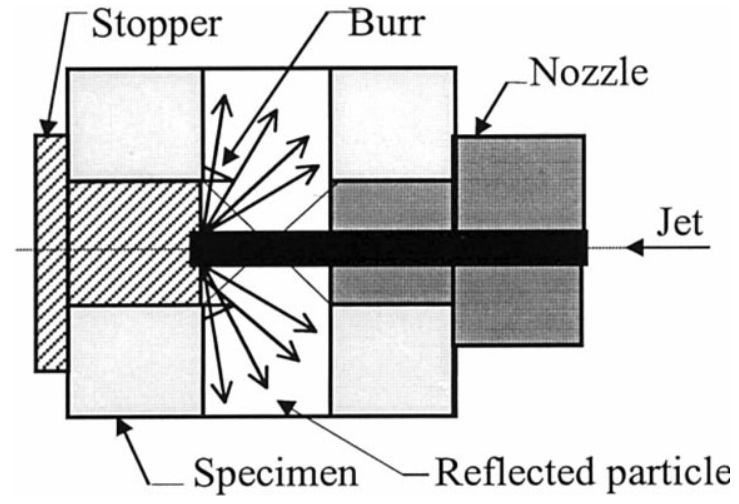

Fig. 3. Deburring set-up.

\section{Experimental design and procedure}

A deburrring experiment constructed using the Taguchi experimental design array $[10,11]$ was performed. The experiments were conducted to identify the factors causing the burr removal and also to determine the effects of each parameter on the deburring process. Four independent parameters, namely SOD, MR, ASIZE and BSD, were varied in the experiments. To produce a fully crossed experimental design array for the two levels of factors, three sets of 16 runs totaling 48 runs were performed. The 16-run experimental design was chosen for its ability to investigate the main effects and the linear two-variable interaction effects. Table 2 shows the level of each variable used in the experiment.

The complete removal of the burr was retained as the response parameter. The output was designated as ' 0 ' or ' 1 '. The value ' 0 ' represents non-removal of the complete burr and ' 1 ' represents removal of the complete burr. Measurements were reduced using the analysis of variance (ANOVA) technique of Taguchi to designate the deburring quality. A software package STATGRAPHICS was used to analyze the data. The percentage effect of each variable was calculated by the ratio of the individual parametric sum of squares to the total sum of squares of all parameters.

The effects of the parameters SOD and MR on deburring process were studied by determining the time to deburr for various SODs at different MR. The effect of the parameter BSD on the deburring process was

Table 2

Level of each variable used in the experiment

\begin{tabular}{lcc}
\hline Variable & Level 1 & Level 2 \\
\hline SOD (mm) & 10 & 40 \\
MR & 0.20 & 0.60 \\
ASIZE grit & 30 & 60 \\
BSD (mm) & 0 & 3 \\
\hline
\end{tabular}


Table 3

The percentage effect of each of the parameters on the process output

\begin{tabular}{lc}
\hline Factor & $\%$ Effect \\
\hline SOD & 14.3 \\
MR & 0 \\
ASIZE & 14.3 \\
BSD & 14.3 \\
Any interaction with MR & 0 \\
Other two-factor interactions & 14.3 \\
\hline
\end{tabular}

studied by determining the amount of material removed from the stopper surface by primary erosion.

\section{Results and discussion}

The results of the ANOVA indicate that the three parameters, SOD, ASIZE and BSD, have a significant effect on the deburring process. Table 3 shows the percentage effect of each of the parameters on the process output. The effects of the two-factor interaction are also shown in Table 3. Deburring was possible only when the levels of the three significant parameters were kept at their lower levels, i.e. when the SOD was 10 $\mathrm{mm}$, the ASIZE was 30 grit and the BSD was $0 \mathrm{~mm}$. The level of MR did not affect the process output. However, further experiments indicated that the deburring time for various SODs changed in different fashions at low and high MRs.

The effects of the SOD and MR on deburring time are shown in Fig. 4. The SOD was varied from 4 to 22 $\mathrm{mm}$. At a lower MR, the deburring time increased continuously with the increase in SOD. This may be due to the fact that as the rebounding particles collide

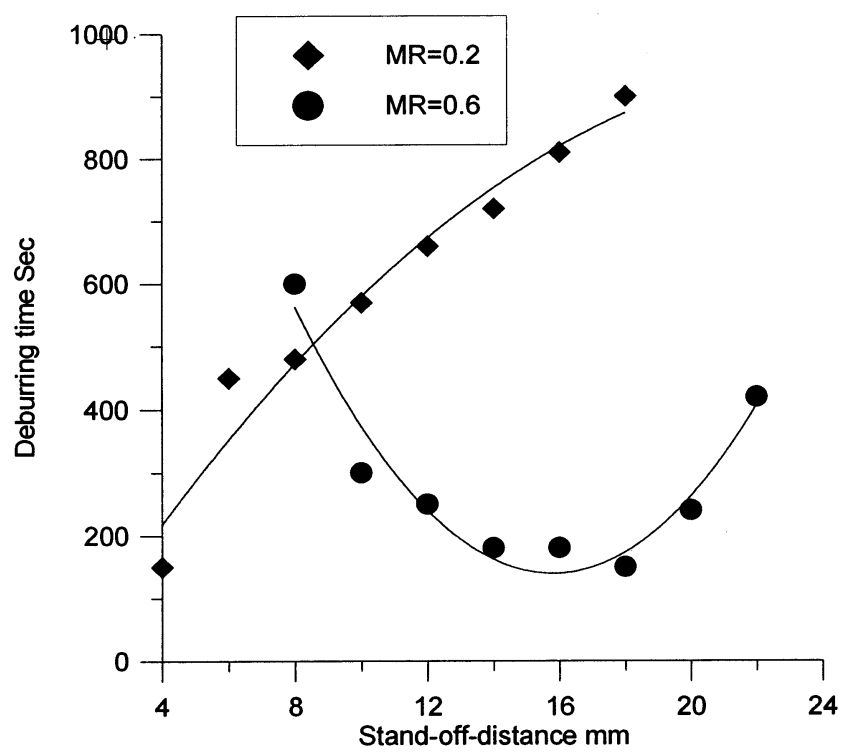

Fig. 4. Deburring time. with the incoming particles, the number of effective particles hitting the burr surface was decreased and this decrement further increased with the increase of SOD. In addition to the collision of the rebounding particles from the stopper surface, the particles are reflected back towards the incoming jet after hitting the burr surface. The restricted passage increased the chances of further collisions with the incoming jet. This multiple collision of the particles with the incoming jet increased with the increasing SOD, and thus, the deburring time increased.

At a higher MR, the deburring time decreased initially with the increasing SOD and after a certain value of SOD, the deburring time started to increase. Owing to increased collision of the particles at lower SOD, the number of effective particles were lesser, giving rise to a longer deburring time; but as the SOD increased up to certain value, the effective number of particles were increased, resulting in a reduced deburring time. After the SOD reached the optimum value, the number of effective particles hitting the burr surface decreased as in the case of lower MR, thus increasing deburring time.

The deburring was effected only when coarser grit abrasive was used. This may be due to the fact that the finer grit particles cause more collisions, resulting in a smaller number of effective particles being available for deburring. In addition, the particles become fragmented during primary impingement. Finer particles become still finer, and become ineffective in removing the material from the burr surface. In case of coarser particles however, the collision might be lesser and therefore the fragmented particles are sufficiently large to remove material from the burr surface.

The burrs were removed only when the BSD parameter was maintained at a value close to $0 \mathrm{~mm}$. At all other values of BSD, pulsating noises were observed during the process and the burrs were not removed. Fig. 5 shows the erosion rate of the stopper owing to primary impingement for various BSD values of crossdrilled holes. The erosion rate was found to decrease with increasing BSD. This may be due to the accumulation of the abrasive particles at the narrow space between the top surface of the stopper and the section where the burr root thickness is maximum. This nonrigid zone of accumulated abrasive particles retards the velocities of the rebound particles and also reduces the number of reflected particles. As the BSD increases, the volume of this zone also increases, resulting in a further decrease in the number of reflected particles and their velocities.

\section{Conclusions}

The results obtained in this investigation support the following conclusions. 


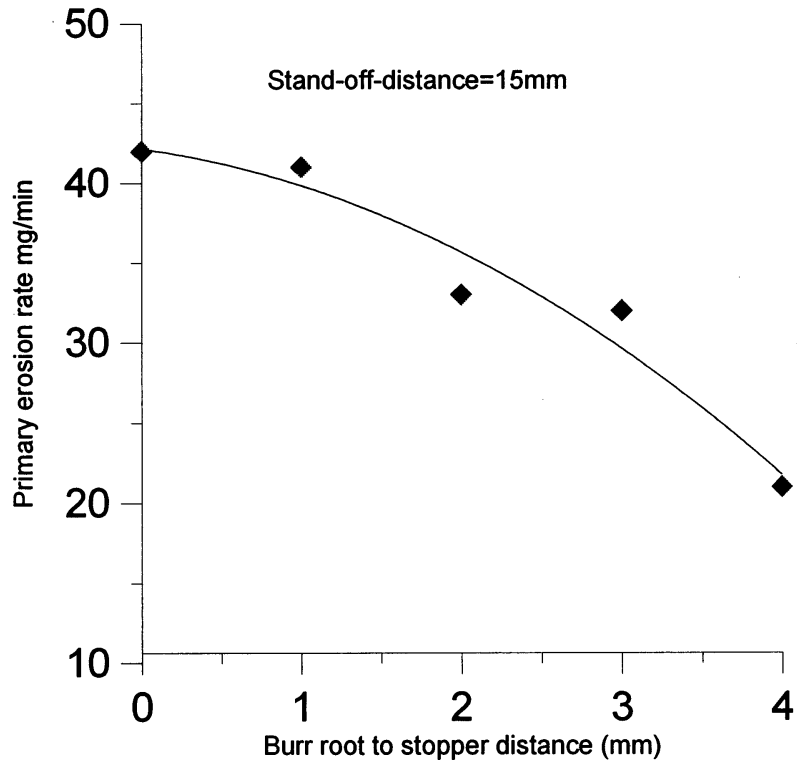

Fig. 5. Effect of BSD on erosion rate.

(i) The abrasive jet deburring of cross-drilled holes with the proposed stopper-type method ensures complete removal of the burrs. The abrasive particles reflected by the stopper remove the burrs by secondary erosion.

(ii) The parameters SOD, ASIZE and BSD and their interactions, affect the deburring process. At lower MR, the deburring time increases with the stand-off distance. In the case of higher MR, the deburring time initially decreases and on reaching an optimum value it increases with the SOD. (iii) Only coarser grit abrasives are effective in deburring.

(iv) Owing to the presence of a non-rigid zone for any value of the BSD parameter other than near to 0 $\mathrm{mm}$, the velocities and the number of effective particles hitting the burr surface decrease and effective deburring is not achieved.

\section{References}

[1] N. Ramachandran, N. Ramakrishnan, Abrasive jet machiningupcoming technology in metal processing - a review, J. Met. Process. Technol. 39 (1) (1993) 21-30.

[2] N Ramachandran, N Ramakrishnan, The role of deburring in manufacturing: a state of survey, J. Mater. Process. Technol. 44 (1994) 1-13.

[3] R.E. Winter, I.M. Hustings, Particle erosion of ductile materials: a mechanism of metal removal, Wear 27 (1974) 121-128.

[4] G.P. Tilly, A two stage mechanism for ductile erosion, Wear 23 (1973) 87-103.

[5] I Finnie, Erosion of surfaces by solid particles, Wear 3 (1960) $87-103$.

[6] Lu Jyh-Woel, A study of the fundamental mechanism of erosion using Hertzian fracture test, Wear 162-164 (1993) 856-863.

[7] G. Sundarajan, G.P. Shewmon, A new model for the erosion of metals at normal incidence, Wear 84 (1983) 237-258.

[8] R. Balasubramaniam, M. Tech. Dissertation, Department of Mechanical Engineering, Indian Institute of Technology, Bombay, 1995.

[9] R. Balasubramaniam, J. Krishnan, N. Ramakrishnan, Some studies on abrasive jet deburring' 17th AIMTDR Conf., Warangal, India, pp. 249-252.

[10] P. Rose, Taguchi Techniques for Quality Management, McGraw Hill, New York, 1988.

[11] Tapan P. Bagchi, Taguchi Methods Explained-Practical Steps to Robust Design, Prentice-Hall, New Delhi, 1993. 